\title{
A criterion for flow mechanisms through vertical sharp-edged orifice and model for the orifice discharge coefficient
}

\author{
Cao Rui, Liu Yansheng* and Yan Chaoyu \\ State Key Laboratory of Heavy Oil Processing, China University of Petroleum, Beijing 102249, China \\ (C) China University of Petroleum (Beijing) and Springer-Verlag Berlin Heidelberg 2011
}

\begin{abstract}
According to the experimental data of the orifice discharge coefficient for the flow through a vertical sharp-edged orifice obtained in the previous study of this work, a theoretical criterion for flow mechanisms of small orifice (viz. thick-walled orifice and nozzle) and large orifice (viz. thin-walled orifice) was proposed based on the ratio of orifice diameter to plate thickness. It can help explain the dissipation of the mechanical energy loss in the flow process for the two flow mechanisms under different operating regimes. The main parameters such as orifice diameter, plate thickness and liquid head were correlated, and a semi-empirical model for orifice coefficient and an empirical model with high precision at the stable region were developed.
\end{abstract}

Key words: Orifice discharge coefficient, theoretical criterion, model, ratio of orifice diameter to plate thickness, Reynolds number, liquid head

\section{Introduction}

Liquid discharging through an orifice is a common example in hydrodynamics. The discharge processes of fluid through a thin-walled orifice or nozzle and the classification of them according to flow characteristics have been reported (Jin, 1994; Luo, 2007; Finnemore and Franzini, 2006). It is found that the liquid attaches to the wall of the channel through the small orifice, but separates from the wall of the channel through the large orifice, when the fluid is turbulent. There is significant difference in the discharge characteristics of the two flow mechanisms. In engineering applications, the orifice discharge coefficient, $C_{0}$, is usually treated as a constant at relatively high liquid head for simplification, since the orifice discharge coefficient approaches to a constant when the Reynolds number, Re, is large enough. The orifice discharge coefficient is usually confirmed at the range of 0.60-0.62 for a thin-walled orifice and 0.82-0.86 for a nozzle. In addition, a number of experiments and computational fluid dynamics (CFD) simulations of orifice discharge are also reported. For example, Zhang and Dong (2000) have studied the orifice flow process of the orifice-typed liquid distributor of a packed tower by investigating the change of the orifice discharge coefficient with different orifice configurations and Reynolds numbers. The equations describing the orifice discharge coefficient were developed by Lanzhou Petroleum Machinery Research Institute (2005) in the calculation of the

*Corresponding author. email: ctray@cup.edu.cn

Received January 5, 2010 pressure drop for sieve tray and dualflow tray. Furthermore, Zhao et al (2004) investigated the profiles of the orifice discharge coefficient for a tapered sieve tray with different perforated angles by experiment and CFD simulation. Li and $\mathrm{Yu}$ (2006) investigated the flow through the bottom orifice of the hanging downcomer in towers. The discharge coefficient for the flow through small sharp-edged cylindrical orifices with a diameter between 0.3 and $2 \mathrm{~mm}$ and an aspect ratio between 1 and 50 were evaluated, and the characteristics of flow in the separated, attached and cavitated flow regions were determined by Ramamurthi and Nandakumar (1999). The pressure drop and void fraction profiles during horizontal flow through thin and thick orifices were experimentally investigated by Fossa and Guglielmini (2002). Borutzky et al (2002) proposed an orifice flow model for laminar and turbulent flow conditions.

Firstly, the empirical data reported in literature (Jin, 1994; Luo, 2007; Finnemore and Franzini, 2006) are quite convenient for engineering applications. However, the fixed orifice discharge coefficient lacks generality in various operating modes, such as for the case at low liquid head or with high turndown. Secondly, the flow patterns of orifice discharge are usually classified based on the ratio of plate thickness to orifice diameter. That is, orifices with $t / d \leq 2$ are classified as thin-walled orifices, ones with $2 \leq t / d \leq 4$ as thickwalled orifices, and the others with $t / d \approx 3 \sim 4$ as nozzles. In general, these studies mainly report the flow characteristics through thin-walled orifices and nozzles. Flow characteristics through thick-walled orifices are rarely reported. In addition, the criterion for the classification of orifice flows is too 
rough in comparison with the experimental data reported in literature (Zhang and Dong, 2000; Ramamurthi and Nandakumar, 1999), and the effects of liquid head, Reynolds Number and physical properties on orifice flow mechanism are not specified. Thirdly, the above mentioned results were obtained for particular orifice dimensions and fluid physical properties and the correlations obtained from these studies are not fit to be extrapolated.

Therefore, the characteristics of $C_{\mathrm{o}}$ for a vertical sharpedged orifice were experimentally investigated in our previous work. According to the distinct profiles of the $C_{\mathrm{o}}$ curves, it was found that the orifice discharge followed the two mechanisms of large orifice (viz. thick-walled orifice) and small orifice (viz. thin-walled orifice and nozzle) (Cao et al, 2008a). Based on the data, the aim of this paper is to establish a theoretical criterion for the flow mechanisms of small orifice and large orifice according to the ratio of the orifice diameter to plate thickness, and to develop a semi-empirical model for the orifice coefficient and a high precision empirical model for a stable flow region, in order to precisely determine the style of orifice flow and to improve the design accuracy of orifice flow process.

\section{Theory criterion for orifice discharge mechanism}

For comparison we assumed that the large orifice has the same diameter as the small orifice, but their plate thickness, $t$, is different (the small orifice with thicker $t$ and the large orifice with thinner $t$ ). According to our previous experimental data and CFD simulated results (Cao et al, $2008 \mathrm{~b}$ ), the mechanical energy loss in the flow region in front of the orifice for a large orifice is nearly the same as that for a small orifice with the same orifice diameter and liquid head (turbulent regime), indicating that the significant difference between the large orifice and small orifice flow mechanisms is caused by the different flow patterns inside the orifice.

In the turbulent regime, the stream could be contracted when flowing through the orifice, and then the stream will be expanded (Wang, 1990). For a small orifice, the expansion of the fluid may occur inside the channel because the channel length is long enough, which restricts the development of the expansion. Therefore, the local energy loss inside the channel is relatively low. However, for a large orifice, the vena contracta occurs out of the channel since the channel length is too short to restrict the expansion of the stream, causing serious separation of the boundary layer and resulting in high mechanical energy loss. Therefore, the location of vena contracta inside or outside the orifice channel can be treated as the theoretical criterion for large orifice and small orifice flow mechanisms. In the laminar flow state, the fluid follows the same flow mechanism for both the large orifice and small orifice. In the transitional region, the turbulent eddies in the liquid do not develop sufficiently. Therefore, the flow parameters in this region can not be treated as the criterion. However, the orifice discharge coefficient $C_{0}$ in the stable turbulent region remains constant. Thus, it is more appropriate to treat the flow parameters in the stable turbulent region as a criterion of critical orifice (Cao et al, 2008a).
The whole flow process through an orifice is similar to that in the Venturi flowmeter (Lu et al, 2004; Liang, 2002) (Fig. 1 ), in which the flow process can be divided into contraction, parallel and expansion regions. During the development of the flow stream, the energy consumed for contraction is far less than that for expansion, which is the main cause of energy loss. The parallel stage is the transition from contraction to expansion and it could be speculated that the stream begins to expand from midway in the parallel region. As a result, a comparison of the sum of the contraction distance $(L)$ and half the parallel flow distance with the channel length $(t)$ could be treated as the criterion for the flow mechanism of the orifice, where the length of the parallel region is approximately equal to the diameter of vena contracta, $d_{\mathrm{c}}$ (Kremlevsky, 1958) (Eq. (1)).

$$
f=t-L-\frac{d_{\mathrm{c}}}{2} \geq 0
$$

If $f \geq 0$ it may be called as small orifice mechanism, yet if $f<0$ it may be called as large orifice mechanism.

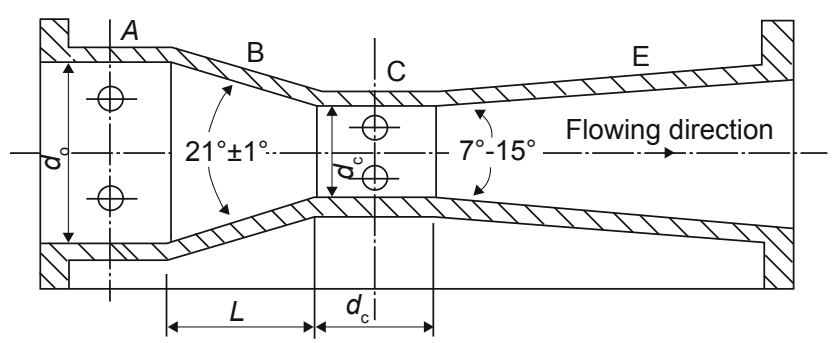

Fig. 1 Sketch of the Venturi flowmeter

According to literature (Kremlevsky, 1958; Jamal, 2004; $\mathrm{Yu}, 2003)$, the contraction distance, $L$, is in the range of about 0.5 to 0.7 . For a fixed orifice configuration, the higher the flow velocity through the orifice, the shorter the contraction distance $(L)$ of the stream. In fact, the contraction distance approaches to $0.5 d$ (orifice diameter) in stable turbulent region because the distribution of flow velocity is confirmed (Finnemore and Franzini, 2006; Sheng, 1980). The distance of parallel region can be calculated according to the following Eq. (2) (Borutzky et al, 2002).

$$
C_{\mathrm{C}}=\frac{\pi}{\pi+2}=0.611
$$

where, $C_{\mathrm{c}}$ is the coefficient of contraction, $C_{\mathrm{c}}=\left(d_{\mathrm{c}} / d\right)^{2}$.

So, the ratio of vena contracta diameter to orifice diameter, $d_{\mathrm{c}} / d$, is about 0.78 . Accordingly, Eq. (3) can be obtained by substituting $L$ and $d_{\mathrm{c}}$ into Eq. (1).

$$
f=t-0.5 d-\frac{0.78 d}{2} \geq 0
$$

Eq. (3) can be transformed as follows.

$$
f=\frac{t}{d}-0.5-\frac{0.78}{2}=\frac{t}{d}-0.89 \geq 0
$$

Eq. (4) is the criterion to definite large orifice (viz. thickwalled orifice, $f<0$ ) and small orifice (viz. thin-walled orifice and nozzle, $f \geq 0$ ). The criterion $f$ was examined by the 
experimental data from our previous work (Cao et al, 2008a). Fig. 2 compared the experimental data and the calculated results from Eq. (4). It can be seen from Fig. 2 that the calculated values of $f$ agree well with the experimental data.

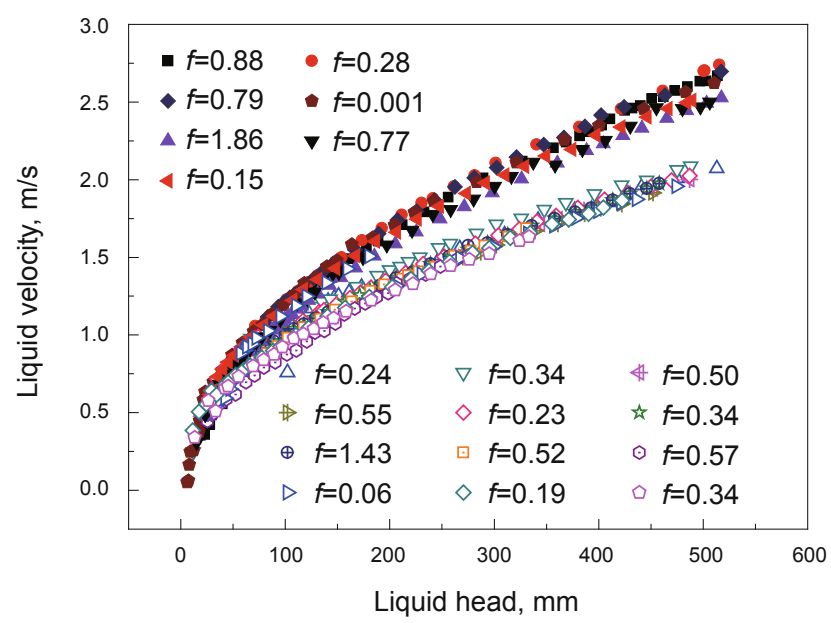

Fig. 2 Criterion $f$ for small and large orifices

\section{Model correlations for orifice discharge}

\subsection{Correlation of orifice discharge coefficient $\left(C_{0}\right)$ with liquid head $(h)$ and configuration dimensions}

According to the previous experimental results (Cao et al, 2008a) and the sketch of orifice flow parameters (Fig. 3), assumptions for the orifice flow were made as follows.

1) The flow region inside orifice includes the halfspherical area with a radius of $d / 2$ and the scope inside the channel;

2) The flow region in front of orifice lies in the halfspherical area with its radius between $0.5 d$ and $k d$ outside the flow region inside the orifice;

3) The contours of the total mechanical energy and physical parameters of the fluid upstream the orifice are a group of concentric spheres and the flow is steady through the orifice;

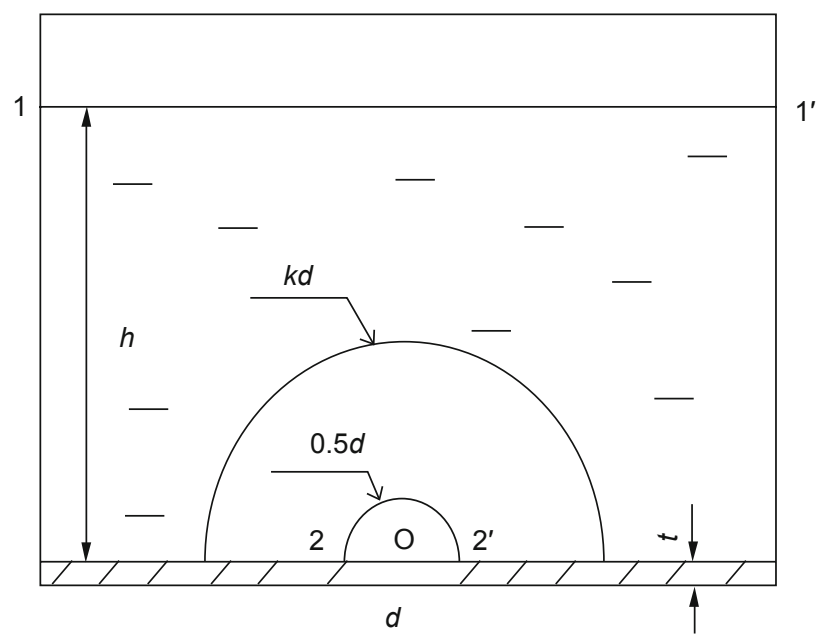

Fig. 3 Sketch of orifice flow parameters
4) The sum of the local energy loss and the friction energy loss on the way is proportional to the square of local velocity.

Bernoulli's equation for section between 1-1' and 2-2' in Fig. 3 is expressed as follows.

$$
\begin{aligned}
& \frac{P_{1}}{\rho}+\frac{u_{1}^{2}}{2}+h g=\frac{P_{2}}{\rho}+\frac{u_{2}^{2}}{2} \\
& +\left(\int_{\frac{1}{2} d}^{k d} \xi_{1}^{\prime}\left(u^{\prime}\right) \frac{u^{\prime 2}}{2} \mathrm{~d} R^{\prime}+\lambda \frac{t+\frac{d}{2}}{d} \frac{u_{2}^{2}}{2}+\xi_{2}\left(u_{2}\right) \frac{u_{2}^{2}}{2}\right)
\end{aligned}
$$

where, $u^{\prime}$ - the liquid velocity at radius of $R^{\prime}\left(k d \geq R^{\prime} \geq 0.5 d\right)$ with spherical center (or circular center) $\mathrm{O}, \mathrm{m} / \mathrm{s}$;

$k$-parameter;

$u_{1}, u_{2}$ - local velocity on section $1-1^{\prime}$ and $2-2^{\prime}, \mathrm{m} / \mathrm{s}$;

$t$-plate thickness, m;

$d$-orifice diameter, $\mathrm{m}$;

$\xi_{1}(u)$-resistance coefficient of the flow region in front of orifice;

$\lambda$-friction resistance coefficient of the flow region inside orifice;

$\xi_{2}(u)$-local resistance coefficient of the orifice outlet;

$P_{1}$ — static pressure on section $1-1^{\prime}, \mathrm{kPa}$;

$P_{2}-$ static pressure on section 2-2', $\mathrm{kPa}$;

$\rho$-density of fluid, $\mathrm{kg} / \mathrm{m}^{3}$

The items in the right square brackets of Eq. (5) are the mechanical energy losses, where the first item is the energy loss in the flow region in front of the orifice, the second is the friction energy loss in the flow region inside the orifice, and the third is the local energy loss at the orifice outlet.

At laminar condition, the flow resistance coefficient of the region in front of the orifice changes slightly with the velocity of fluid, so it can be expressed as $\xi_{1}(u)=C$ (where $C$ is an constant) for the purpose of simplification. At turbulent condition, the coefficient increases sharply with increasing velocity, and it can be expressed as $\xi_{1}(u)=C u^{2}$.

The continual equation, $\frac{\pi}{2} d^{\prime 2} u^{\prime}=\frac{\pi}{2} d^{2} u_{d / 2}=\frac{\pi}{4} d^{2} u_{2}$, can be expressed as $u^{\prime}=\frac{d^{2}}{d^{\prime 2}} u_{d / 2}=\frac{d^{2}}{2 d^{\prime 2}} u_{2}$. Let $d^{\prime}=2 R^{\prime}$, in case of $u^{\prime}=0.01 u_{2}$, the maximum range of the flow region in front of the orifice (Cao et al, 2008b), $d^{\prime}{ }_{\max }$, is calculated as follows:

$$
\frac{u^{\prime}}{u_{d / 2}}=\frac{u^{\prime}}{2 u_{2}}=\frac{\pi d^{2} / 2}{\pi d^{\prime 2} / 2}=50 \Rightarrow d_{\text {max }}^{\prime}=7 d
$$

Therefore, the energy loss under laminar conditions in the flow region in front of the orifice can be calculated as follows.

$$
\begin{aligned}
\int_{\frac{1}{2} d}^{k d} \xi_{1}\left(u^{\prime}\right) \frac{u^{\prime 2}}{2} \mathrm{~d} R^{\prime} & =C_{1}^{\prime} \int_{\frac{1}{2} d}^{k d} \frac{\left(\frac{u_{2} d^{2}}{2 d^{\prime 2}}\right)^{2}}{2} \mathrm{~d} R^{\prime} \\
& =C_{1}^{\prime} \frac{u_{2}^{2} d^{4}}{2^{7} \times 3}\left(\frac{2^{3}}{d^{3}}-\frac{1}{7^{3} d^{3}}\right)=C_{1} d u_{2}^{2}
\end{aligned}
$$


The energy loss in turbulent conditions of that flow region is calculated as follows.

$$
\begin{aligned}
\int_{\frac{1}{2} d}^{k d} \xi_{1}\left(u^{\prime}\right) \frac{u^{\prime 2}}{2} \mathrm{~d} R^{\prime} & =C_{1}^{\prime} \int_{\frac{1}{2} d}^{k d} \frac{\left(\frac{u_{2} d^{2}}{2 d^{\prime 2}}\right)^{4}}{2} \mathrm{~d} R^{\prime} \\
& =C_{1}^{\prime} \frac{u_{2}^{4} d^{8}}{2^{13} \times 7}\left(\frac{2^{7}}{d^{7}}-\frac{1}{7^{7} d^{7}}\right)=C_{1} d u_{2}^{4}
\end{aligned}
$$

Since $u_{1} \approx 0$ and $P_{1}=P_{2}$, Bernoulli's equation is simplified as follows.

$$
h g=\frac{u_{2}^{2}}{2}+\left(\xi_{1}\left(u_{2}\right) u_{2}^{2}+\lambda \frac{t+\frac{d}{2}}{d} \frac{u_{2}^{2}}{2}+\xi_{2}\left(u_{2}\right) \frac{u_{2}^{2}}{2}\right)
$$

\subsubsection{Correlations for small orifice}

For a small orifice, the local resistance at the outlet can be ignored. The local energy loss due to the expansion is relatively low, and can be incorporated in the friction resistance inside the orifice.

$$
h g=\frac{u_{2}^{2}}{2}+\left(\xi_{1}\left(u_{2}\right) u_{2}^{2}+\lambda \frac{t+\frac{d}{2}}{d} \frac{u_{2}^{2}}{2}\right)
$$

\section{Laminar flow}

For laminar flow $(\operatorname{Re}<2300)$, based on the conventional equation for pipe flow of $\lambda=64$, the friction resistance coefficient is expressed as $\lambda=\frac{C}{u d}$. Then Eq. (9) can be expressed as follows.

$$
h g=\frac{u_{2}^{2}}{2}+\left(C_{1} d u_{2}^{2}+C_{2} \frac{t+\frac{d}{2}}{d^{2}} \frac{u_{2}}{2}\right)
$$

The coefficients of the correlation simulated from experimental results are: $C_{1}=22.47 ; C_{2}=5.15 \times 10^{-4}$.

Then, $C_{\mathrm{o}}$ is calculated according to Eq. (10):

$$
C_{o}=Q / A_{0} \sqrt{2 g h}
$$

The average relative error of $C_{\mathrm{o}}$ between the experimental data and the calculated values is $4.06 \%$, with the maximum relative error of $15.34 \%$. Fig. 4 shows the comparison of experimental data and fitting values at laminar condition for small orifice.

\section{Turbulent flow}

For turbulent flow $(R e \geq 2300)$, based on the conventional equation for pipe flow of $\lambda=0.1\left(\frac{e}{d}+\frac{68}{R e}\right)^{0.23}$, the friction resistance coefficient is expressed as $\lambda=C_{2}\left(\frac{C_{3}}{d}+\frac{C_{4}}{u d}\right)^{C_{5}}$, Then Eq. (9) can be written as follows.

$$
h g=\frac{u_{2}^{2}}{2}+\left(C_{1} d u_{2}^{4}+C_{2}\left(C_{3}+\frac{C_{4}}{u}\right)^{C_{5}} \frac{t+\frac{d}{2}}{d^{2}} \frac{u_{2}^{2}}{2}\right)
$$

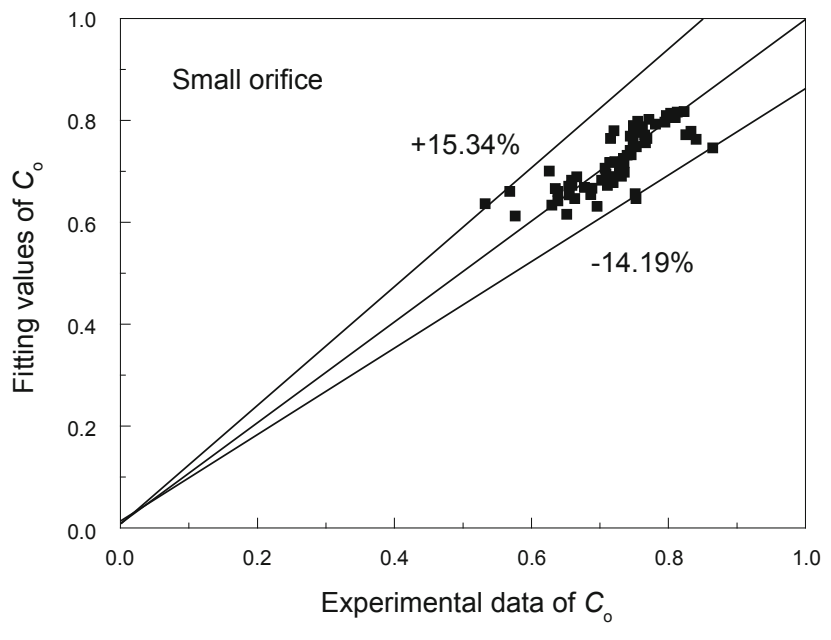

Fig. 4 Analysis of error $(f>0, \operatorname{Re}<2300)$

The coefficients of the correlation are listed below. $C_{1}=0.0010, C_{2}=0.0974, C_{3}=0.2183, C_{4}=0.0746, C_{5}=0.1758$

The average relative error of $C_{\mathrm{o}}$ between the experiment results and the fitting results is $3.17 \%$, with the maximum value of $15.06 \%$. Fig. 5 shows the comparison of experimental data and fitting values at turbulent condition for

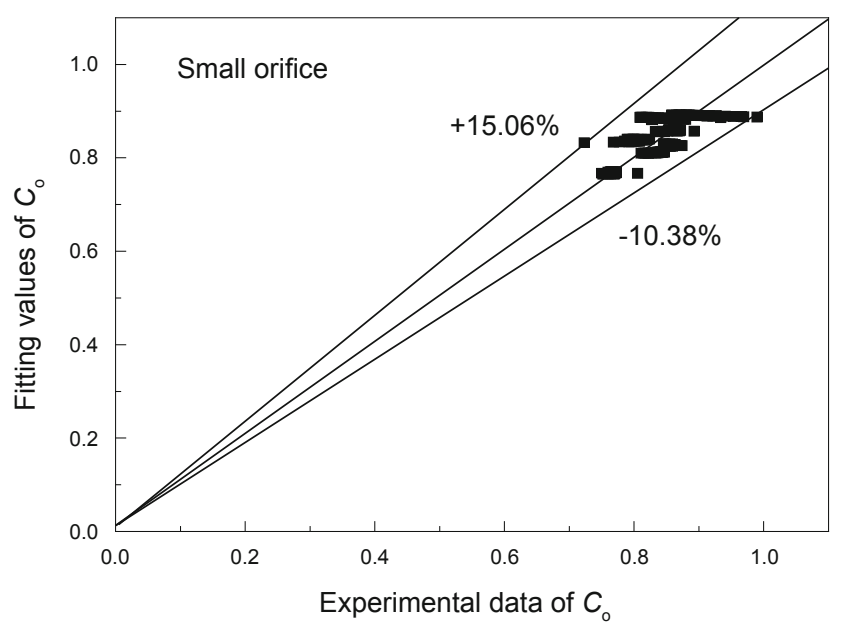

Fig. 5 Analysis of error $(f>0, R e \geq 2300)$

small orifice.

\subsubsection{Correlations for large orifices}

According to the experimental data, the orifice flow is basically in laminar condition in case of $h<50 \mathrm{~mm}$, while in turbulent condition in case of $h \geq 50 \mathrm{~mm}$. Therefore, $h=50 \mathrm{~mm}$ can be assumed to be the critical point in the model (Cao et al, 2008a).

\section{Laminar flow}

According to the large orifice, the model patterns have no essential difference from a small orifice in laminar conditions except that the range of the flow region in front of orifice is slightly larger. Therefore, the $d / t$ is introduced in the Eq. (12) below.

$$
h g=\frac{u_{2}^{2}}{2}+\left(C_{1}\left(\frac{d}{t}\right) d u_{2}^{2}+\frac{C_{2}}{d^{2}}\left(t+\frac{d}{2}\right) \frac{u_{2}}{2}\right)
$$

The coefficients of the correlation are simulated as: 
$C_{1}=2.76, C_{2}=0.002181$.

The average relative error of $C_{\mathrm{o}}$ between the experiment results and the fitting results is $4.68 \%$, with the maximum value of $16.63 \%$. Fig. 6 shows the comparison of experiment data and fitting values at laminar condition for large orifice.

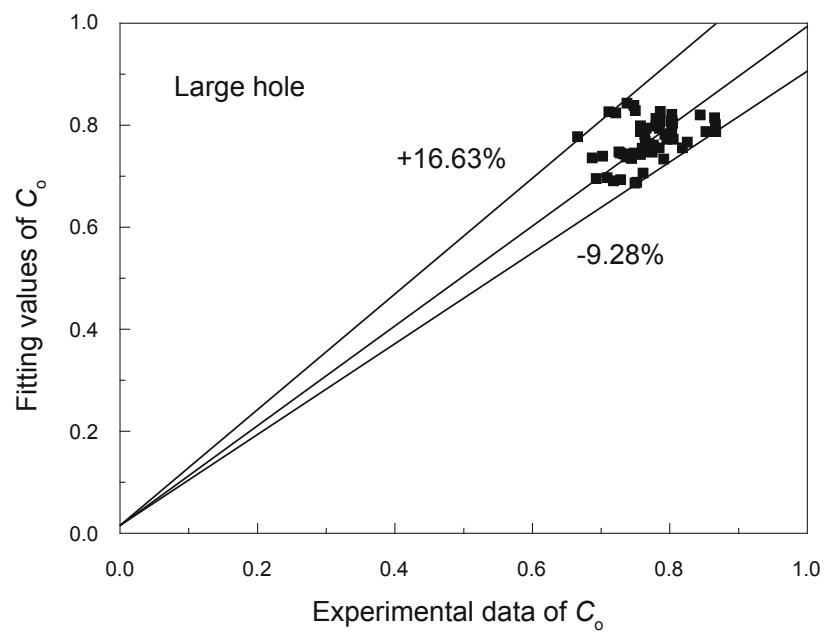

Fig. 6 Analysis of error $(f>0, h<50 \mathrm{~mm})$

\section{Turbulent flow}

When the flow is turbulent the friction may be ignored due to the short channel, but the local resistance in the outlet is dominant, especially in a stable regime, where the sum of the resistance coefficient approaches to a constant. Therefore, it can be expressed by Eq. (13).

$$
h g=\frac{u_{2}^{2}}{2}+\left(C_{1} d u_{2}^{4}+C_{2} \exp \left(C_{3} \frac{d}{t}\right) \frac{u_{2}^{2}}{2}\right)
$$

The coefficients of the correlation are listed below. $C_{1}=6.80, C_{2}=1.038, C_{3}$

The average relative error of $C_{\mathrm{o}}$ between the experiment results and the fitting results is $3.46 \%$, with the maximum value of $16.45 \%$ (Fig. 7 ).

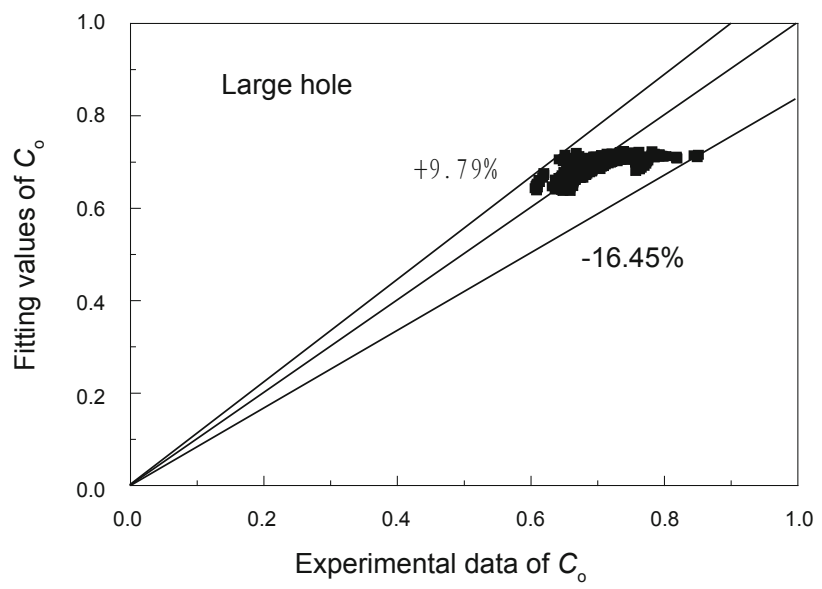

Fig. 7 Analysis of error $(f>0, h \geq 50 \mathrm{~mm})$

\subsection{Correlation of $C_{0}$ in stable regime}

In terms of the orifice flow in stable regime widely used in practical applications, the above correlations are not accurate enough. Therefore, a new experiential correlation is brought forwards as shown in Eq. (14).

$$
C_{o}=1-C_{1} \frac{\exp \left(C_{2} d^{C_{3} t}\right)}{t^{C_{4}}}-\frac{C_{5}}{d^{2}}
$$

If $d \leq 1.2 t, C_{1}=2.01 ; \quad C_{2} \quad \square \square C_{3}=2.85 ; C_{4}=0.174 ;$ $C_{5}=0.0005$

If $d>1.2 t, C_{1}=6.01 ; \quad C_{2}=5.79 ; \quad C_{3}=4.93 ; \quad C_{4}$ $C_{5}=0.0002$

If $d \leq 1.2 t$, the average relative error of Eq. (14) is $0.48 \%$, with a maximum value of $0.91 \%$. If $d>1.2 t$, the average relative error is $1.31 \%$, with the maximum value of $2.77 \%$. Fig. 8 shows the comparison of experiment data and fitting values in stable region.

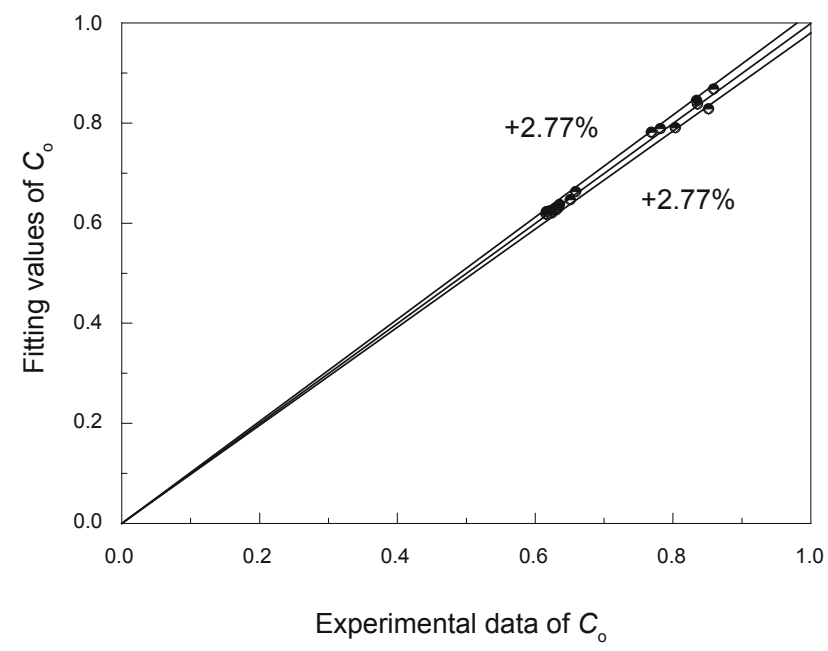

Fig. 8 Analysis of error

\section{Conclusions}

(1) Base on the orifice flow mechanism, the equation of the theoretical criterion is introduced to define the large orifice (viz. thick-walled orifice) and small orifice (viz. thin-walled orifice and nozzle), according to the ratio of orifice diameter to plate thickness. If $f \geq 0$, the flow obeys the mechanism of small orifice; if $f<0$, the flow obeys the mechanism of large one. The criterion has been verified by the experimental data of the previous work. It can be found that the calculated values of $f$ criterion agree well with the experimental data.

(2) Base on the experimental data, two semi-empirical correlations of $C_{\mathrm{o}}$ are proposed for the small orifice and large orifice in laminar and turbulent conditions, respectively. In addition, the empirical correlations with high precision at stable regime are established, which are recommended for use in relevant industrial design process.

\section{Acknowledgements}

This work was supported by the National Natural Science Foundation of China (20806090).

\section{References}

Borutzky W, Barnad B and Thoma J. An orifice flow model for laminar 
and turbulent conditions. Simulation Modelling Practice and Theory. 2002. (10): 141-152

Cao R, Liu Y S, Yan C Y, et al. Characteristics of the vertical sharpedged orifice discharge (I): Effect of flow regime and configuration parameters on orifice discharge coefficient. Journal of Chemical Industry and Engineering (China). 2008a. 59(9): 2175-2180 (in Chinese)

Cao R, Liu Y S, Yan C Y, et al. Characteristics of vertical sharp-edged orifice discharge (II): Behavior of fluid in contributing flow region in front of orifice. Journal of Chemical Industry and Engineering (China). 2008b. 59(11): 2741-2749 (in Chinese)

Finnemore E J and Franzini J B. Fluid Mechanics with Engineering Applications. Beijing: Mechanical Industry Press. 2006. 381-388 (in Chinese)

Fossa M and Guglielmini G. Pressure drop and void fraction profiles during horizontal flow through thin and thick orifices. Experimental Thermal and Fluid Science. 2002. 26: 513-523

Jamal M S. Fluid Flow Handbook. Beijing: China Petrochemical Press. 2004. 149 (in Chinese)

Jin Z M. Hydraulic Pressure Fluid Mechanics. Beijing: National Defense Industry Press. 1994. 206-213 (in Chinese)

KremlevskуЛЛ. Flowmeter. Beijing: Hydraulic and Electrical Engineering Press. 1958. 27-29 (in Chinese)

Lanzhou Petroleum Machinery Research Institute. Contemporary Tower Technology (Second Edition). Beijing: China Petrochemical Press. 2005. 236-238, 503-508 (in Chinese)
Li Y M and Yu X M. Study of vapor-liquid flow states of hanging downcomer bottom-orifices. Chemical Engineering. 2006. 34(10): 2426, 62 (in Chinese)

Liang G W. Flow Measurement Technology and Instruments. Beijing: Mechanical Industry Press. 2002. 47-51 (in Chinese)

Lu H Q. Theory and Application of Jetting technology. Wuhan: Wuhan University Press. 2004. 59-74 (in Chinese)

Luo T Q. Fluid Mechanics. Beijing: Mechanical Industry Press. 2007. 74-78 (in Chinese)

Ramamurthi K and Nandakumar K. Characteristics of flow through small sharp-edged cylindrical orifices. Flow measurement and Instrumentation. 1999. (10): 133-143

Sheng J C. Hydraulic Pressure Fluid Mechanics. Beijing: Mechanical Industry Press. 1980. 172-199 (in Chinese)

Wang Z Q. Viscous Fluid Mechanics. Haerbin: Haerbin Industry University Press. 1990. 233-235 (in Chinese)

Yu G C. Chemical Mechanical Engineering Handbook (Volume I). Beijing: Chemistry Industry Press. 2003. 3-56 (in Chinese)

Zhang J C and Dong Y R. Experiment to the orifice discharge coefficient of orifice-typed liquid distributor of packed tower. Chemical Engineering. 2000. 28(3): 10-12, 48 (in Chinese)

Zhao X X, Yao K J, Wang L H, et al. Experimental study and CFD numerical simulation on discharge coefficients of tapered sieve tray. Petrochemical Technology. 2004. 33(5): 428-431 (in Chinese)

(Edited by Zhu Xiuqin) 